\title{
Imaging of meibomian glands: from bench to bedside and back
}

\author{
Edoardo Villani ${ }^{1,2,3} \cdot$ Reiko Arita $^{3,4}$
}

Received: 7 January 2019 / Accepted: 21 January 2019 / Published online: 6 February 2019

(c) The Royal College of Ophthalmologists 2019

In this issue of Eye, Randon et al. [1] present the interesting findings of an in vivo confocal microscopy (IVCM) crosssectional study in which they describe different patterns of presumed meibomian glands (MGs) abnormalities and propose a new IVCM-meibomian gland dysfunction (MGD) classification. This research represents an interesting effort toward a personalized diagnostic and therapeutic approach to MGD patients.

In the last years, we have seen an imaging revolution in the study of MGD. Technological evolution provided us new non- (or nearly non-) invasive tools, including infrared (IR) meibography [2], IVCM, and optical coherence tomography meibography (OCT-M) [3, 4]. The availability of a large amount of novel morphological information accompanied the recent development of our knowledge of MGs physiopathology and the growing awareness of the crucial role of MGs for the ocular surface health and disease [5, 6].

Meibography is the approach with the longest history and the highest level of evidence and IR meibography is an important evolution, allowing non-invasive examination, without the contact of an illuminating probe with the patient's skin $[2,7,8]$. This technique, based on the high reflectivity of healthy MGs, allows to visualize the wholetarsal plate and to quantify the area of MGs drop-out (meiboscore) [2] (dark areas).

Several groups used this technique to assess MGD patients and to describe MGs changes associated to different conditions, including aging [2], contact lens wear, ocular allergy, and severe inflammatory ocular surface diseases $[7,8]$.

IR meibography showed promising results a diagnostic test for MGD, with sensitivity and specificity of $49.3 \%$ and $64.5 \%$, respectively, if used as a single test, and excellent diagnostic accuracy if used in combination with clinical

Edoardo Villani

edoardo.villani@unimi.it

1 Department of Clinical Science and Community Health, University of Milan, Milan, Italy and/or functional examinations [2, 7, 8]. It's increasingly used in clinical practice for MGD diagnosis and follow-up and it has been included in clinical trials as a surrogate endpoint. Moreover, the development of software for automated measurement of meibomian gland area [9] has facilitated evaluation of the efficacy of various treatments of MGD patients. Improvement of user-friendly digital analysis software is likely to further promote the application of IR meibography in clinical practice.

Main limitations of IR meibography are the relatively low resolution, inadequate to show micro-anatomical details and inflammatory cells, and its two-dimensional approach, unable to provide depth information. Although, IR meibography itself is objective and repeatable, interpretation of the resulting images remains subjective. In other words, it's still unclear the exact meaning of the "dark drop-out areas", which might reflect a complete loss of MGs or loss or degeneration of lipid content within a relatively intact gland structure [7].

The recently developed OCT-M, using a customized system with a long coherence swept laser source with a wavelength of $1300 \mathrm{~nm}$, seems to be able to provide interesting 3-D information, helping to discriminate different structural changes corresponding to the IR "dark drop-out areas" $[4,10,11]$. However, at present, we have just preliminary data obtained with customized systems.

IVCM is an emerging nearly non-invasive technology that allows us to see ocular surface structures at the cellular level, providing images with magnification and resolution comparable to histological methods without significantly affecting the steady state of the target tissues [12-14].

The laser scanning IVCM has the ability to go beyond the cornea and to examine the different components of the

2 Eye Clinic San Giuseppe Hospital, IRCCS Multimedica, Milan, Italy

3 Lid and Meibomian Gland Working Group (LIME), Tokyo, Japan

4 Itoh Clinic, Saitama, Japan 
ocular surface, opening new doors for studying the physiology and pathology of this complex morpho-functional unit [12-14].

The first confocal images of presumed MGs were published in November 2005 by Kobayashi et al. [15]. In the last years, Asian and European research groups explored this application of IVCM [12-14], describing several quantitative parameters, with sensitivity and specificity for MGD diagnosis ranging from 80 to $100 \%[14,16]$, and reporting quantitative and qualitative MGs changes related to several conditions, including aging, contact lens wear, ocular allergy, and severe ocular surface inflammatory diseases [14]. Interestingly, IVCM provided the first in vivo evidence in humans of primary atrophic age-related MGD, in contrast with the "hyperkeratinization-obstruction-dilatation" theory [17]. Moreover, this technology allows to analyze the micro-anatomy of the tissue, including the density of inflammatory cells and the presence of fibrosis.

Together with the limited availability and the lack of validated and user-friendly software for images analysis, major limitations of IVCM are the small field of view $\left(<0.25 \mathrm{~mm}^{2}\right)$ and the analysis purely based on morphology and reflectivity. The lack of tissue and cellular phenotyping raises several concerns on images interpretation and on the correlations between confocal and histologic findings.

A recent research by Zhou and Robertson [18] tried to compare IVCM and immunoflorescence findings. These authors concluded that confocal images previously reported as MGs acini should be interpreted as rete ridges in the eyelid dermal-epidermal junction. This hypothesis leaves many unanswered questions and it will require further studies in the next future.

In conclusion, the ongoing imaging revolution in MGs assessment is teaching us a lot on these glands' physiopathology and it's stimulating a virtuous cycle of increase of knowledge. Moreover, we now have some tools (as IR meibography) ready to play an important role in the diagnosis and follow-up of MGD patients, and new improved tools promise to help to move toward a more personalized approach to the patient. Finally, wider inclusion of imaging biomarkers and surrogate endpoints in clinical trials on MGD might be essential to obtain quicker, cheaper, and more successful clinical studies. On the other hand, major efforts are needed and a lot of work is still to do to improve the validation of these tools and to avoid the potential consequences of biomarker misuse or of the choice of invalid surrogate endpoints [19].

\section{Compliance with ethical standards}

Conflict of interest E.V. declares no potential conflict of interest. R.A. holds patents on a non-contact meibography technique (Japanese patent registration no. 5281846; U.S. patent publication no. 2011-
0273550A1; European patent publication no. 2189108A1) and the meibomian gland analysis software (Japn5856385 and US9320439). R.A is applying for the patent of the meibomian gland analysis software for Europe (EP2695570A).

Publisher's note: Springer Nature remains neutral with regard to jurisdictional claims in published maps and institutional affiliations.

\section{References}

1. Randon M, Aragno V, Abbas R, Hong L, Labbé A, Baudouin C. In vivo confocal microscopy classification in the diagnosis of meibomian gland dysfunction. Eye. 2018. https://doi.org/10.1038/ s41433-018-0307-9. [Epub ahead of print]

2. Arita $R$, Itoh $K$, Inoue $K$, Amano $S$. Noncontact infrared meibography to document age-related changes of the meibomian glands in a normal population. Ophthalmology. 2008;115:911-5.

3. Geerling G, Baudouin C, Aragona P, et al. Emerging strategies for the diagnosis and treatment of meibomian gland dysfunction: proceedings of the OCEAN group meeting. Ocul Surf. 2017;15:179-92.

4. Yoo YS, Na KS, Byun YS, et al. Examination of gland dropout detected on infrared meibography by using optical coherence tomography meibography. Ocul Surf. 2017;15:130-8.e1.

5. Chhadva P, Goldhardt R, Galor A. Meibomian gland disease: the role of gland dysfunction in dry eye disease. Ophthalmology. 2017;124:S20-6.

6. Baudouin C, Messmer EM, Aragona P, et al. Revisiting the vicious circle of dry eye disease: a focus on the pathophysiology of meibomian gland dysfunction. Br J Ophthalmol. 2016;100:300-6.

7. Arita R. Meibography: a Japanese perspective. Invest Ophthalmol Vis Sci. 2018;59:DES48-55.

8. Arita R, Fukuoka S, Morishige N. New insights into the morphology and function of meibomian glands. Exp Eye Res. 2017;163:64-71.

9. Arita R, Suehiro J, Haraguchi T, Shirakawa R, Tokoro H, Amano $\mathrm{S}$. Objective image analysis of the meibomian gland area. $\mathrm{Br} \mathrm{J}$ Ophthalmol. 2014;98:746-55.

10. Hwang HS, Shin JG, Lee BH, et al. In vivo 3D meibography of the human eyelid using real time imaging fourier-domain OCT. PLoS ONE. 2013;8:e67143.

11. Liang Q, Pan Z, Zhou M, et al. Evaluation of optical coherence tomography meibography in patients with obstructive meibomian gland dysfunction. Cornea. 2015;34:1193-9.

12. Villani E, Baudouin $\mathrm{C}$, Efron $\mathrm{N}$, et al. In vivo confocal microscopy of the ocular surface: from bench to bedside. Curr Eye Res. 2014;39:213-31.

13. Villani E, Magnani F, Viola F, et al. In vivo confocal evaluation of the ocular surface morpho-functional unit in dry eye. Optom Vis Sci. 2013;90:576-86.

14. Matsumoto Y, Ibrahim OMA. Application of in vivo confocal microscopy in dry eye disease. Invest Ophthalmol Vis Sci. 2018;59:DES41-7.

15. Kobayashi A, Yoshita T, Sugiyama K. In vivo findings of the bulbar/palpebral conjunctiva and presumed meibomian glands by laser scanning confocal microscopy. Cornea. 2005;24:985-8.

16. Ibrahim OM, Matsumoto Y, Dogru M, et al. The efficacy, sensitivity, and specificity of in vivo laser confocal microscopy in the diagnosis of meibomian gland dysfunction. Ophthalmology. 2010;117:665-72.

17. Villani E, Canton V, Magnani F, Viola F, Nucci P, Ratiglia R. The aging meibomian gland: an in vivo confocal study. Invest Ophthalmol Vis Sci. 2013;54:4735-40. 
18. Zhou S, Robertson DM. Wide-field in vivo confocal microscopy of meibomian gland acini and rete ridges in the eyelid margin. Invest Ophthalmol Vis Sci. 2018;59:4249-57.
19. Villani E, Massaro D, Scaramuzzi M, Hamrah P, Medeiros FA, Nucci P. Decade-long profile of imaging biomarker use in ophthalmic clinical trials. Invest Ophthalmol Vis Sci. 2017;58:BIO76-81. 